\title{
CARDIOTOXIC EFFECTS OF VENOMS FROM Chironex fleckeri AND Chiropsalmus sp. ON AN INVERTEBRATE MODEL
}

\section{CARRETTE T. (1), SEYMOUR J. (1)}

(1) Department of Tropical Biology, James Cook University, Australia.

ABSTRACT: Extracted venoms from two species of cubozoan, Chironex fleckeri ( $C$. fleckeri) and Chiropsalmus sp., were injected into the freshwater crayfish Cherax quadricarinatus (C. quadricarinatus). By means of a Vascular Doppler, the cardiac parameters heart rate and contraction amplitude were recorded, with the theoretical total heart output calculated from these two recordings. Cherax quadricarinatus reflects prey items utilized by these two species in the wild and so comparisons of venom action on this animal have greater ecological relevance than those of previous mammalian models used. While the results of this study reflect previous research showing that the overall potency of $C$. fleckeri venom is greater than that of Chiropsalmus sp. venom, the action of the venoms on the heart muscle is found to be different. Chironex fleckeri, which has the most potent venom out of these two species, is actually less effective in reducing cardiac activity than Chiropsalmus sp., which may be a direct reflection of the variation in primary prey utilized by each species.

KEY WORDS: cubozoan, venom, cardiac function, Cherax quadricarinatus, Chironex fleckeri, Chiropsalmus sp.

\section{CORRESPONDENCE TO:}

TERESA CARRETE, Department of Tropical Biology, James Cook University, McGregor Road, Cairns, 4878, Australia. Phone: +61 74042 1724. Fax: +60 74042 1319. Email: teresa.carrette@jcu.edu.au. 


\section{INTRODUCTION}

Previous studies into venom from Chironex fleckeri have shown cardiac effects such as bradycardia produced in an assortment of predominantly vertebrate models (10, $11,13)$, with comparable bradycardia having also been reported for Chiropsalmus sp. venom, likewise tested on vertebrate models (13).

Separated venom fractions from both species have been shown to elicit varying effects on both vertebrate models and isolated invertebrate muscle preparations $(9$, $10,11,12,13)$, however, no direct comparisons of these two complete venom preparations have been conducted, particularly in reference to prey type targeted by these species. Previous cardiac toxicity trials have been based predominantly on rats, mice and guinea pig models $(10,11,12,17)$ and although these experimental animals may be relevant for insight into human envenoming, they do not feature as part of the natural predatory habits of cubozoans. In fact, for all the research into the venom of cubozoans, no study has investigated the changes that occur in the cardiac muscles of a relevant prey type after envenoming.

This study aims to investigate the venom from mature specimens of both Chironex fleckeri and Chiropsalmus sp. on a whole invertebrate animal model similar to prey items utilized by both species in the wild. It serves to examine the venom action from an ecological perspective and compare this to previous venom research predominantly focused on venom actions on terrestrial vertebrates and isolated muscle preparations.

\section{MATERIALS AND METHODS}

There are only two identified species of common large, multi-tentacled cubomedusae (Chiropsalmus quadrigatus and Chironex fleckeri) from the family Chirodropidae in Australian waters (1). The original description of $C$. quadrigatus reports the gonads to be 'cox comb' in shape (14). Chiropsalmus sp. present in Australia does not have 'cox comb' gonads but has a cluster of eight grape-like gonads within the bell, suggesting it is probably a different species from that originally described by Haeckel. Therefore the cubozoan usually termed $C$. quadrigatus in Australia is referred to as Chiropsalmus sp. in this study.

Mature specimens of Chironex fleckeri and Chiropsalmus sp. were collected from Mission Beach and Palm Cove in North Queensland with the tentacles of the animals 
removed and their nematocysts extracted and lyophilized as per Bloom (3). Venom from lyophilized nematocysts was extracted as per Carrette and Seymour (5). The highest concentration of venom used for both species caused death in less than 1 minute while the lowest concentrations used still resulted in death of experimental animals in less than 7 minutes. These concentrations were selected to cover the range of real time to death of prey by naturally envenomed animals based on field observations.

Cardiac effects of extracted venom were examined on the freshwater crayfish Cherax quadricarinatus. Venom was intramuscularly injected into the $2^{\text {nd }}$ abdominal segment of $C$. quadricarinatus specimens, and sound files of heart activity were collected via a vascular Doppler and Avisoft SAS lab Pro software (6).

Sound files of heart activity, recorded using a Vascular Doppler, were collected for an initial period of 20 seconds before the needle was introduced for injection of venom. Heart activity recorded in this first 20 seconds was used as a control period with which cardiac activity after venom injection was compared. Heart rate was measured as beats per 20-second intervals from the start of experimentation until time of cardiac death. Cardiac amplitude was measured as the height of the sound file peaks (6) and again was recorded at each consecutive 20-second interval until asystole. Total cardiac output (heart rate $\mathrm{x}$ heart amplitude) was additionally calculated from these two measurements.

The initial relative heart rates and cardiac amplitudes of $C$. quadricarinatus varied between animals, so data were transformed to allow for comparisons. The initial 20 seconds of cardiac activity before injection (control period) was defined as initial heart rate and amplitude and set at $100 \%$. Each subsequent recording was then converted to a percentage of this initial control period. These transformed data were then combined to calculate the theoretical total heart output over a 20-second interval.

As time to death between experimental animals also varied with venom concentrations, time to cardiac standstill was also converted to a percentage. Actual time of cardiac death was seen as a time period of $100 \%$ with each proceeding time interval calculated as a percentage of this. These data points, recorded as percentages, were then grouped into time categories ranging from one to ten (category $1: 0 \%-10 \%$, category $2: 10 \%-20 \%$, etc.) to allow for comparative analysis. 
These data transformations allowed trends of heart rate, amplitude and output to be directly compared independent of venom concentration, species of cubozoan used, and individual cardiac activity of experimental animals.

\section{RESULTS}

A larger dosage of venom was required to produce a similar time of death in the test animals Cherax quadricarinatus when using Chiropsalmus sp. venom as compared to Chironex fleckeri venom (Figure 1). However, the heart rate of injected $C$. quadricarinatus decreased more rapidly with the introduction of Chiropsalmus sp. venom than after injection of $C$. fleckeri venom (Figure 2). Just prior to death, heart rates of animals injected with $C$. fleckeri venom were approximately $75 \%$ of their original rate compared to the $50 \%$ decrease in heart rate for animals injected with Chiropsalmus sp. venom. Chiropsalmus sp. venom also had a more pronounced effect on cardiac amplitude with test animals recording only $6 \%$ of initial amplitude just prior to death compared to $23 \%$ for animals injected with $C$. fleckeri venom (Figure 3).

Total theoretical cardiac output, as calculated from rate and amplitude, reflected this trend. Just prior to death, after $C$. fleckeri venom injection, the theoretical percentage of total heart output for $C$. quadricarinatus was calculated to be approximately $12 \%$ of its original level compared to animals injected with Chiropsalmus sp. venom, which are at an estimated $1 \%$ of their original level (Figure 4). 


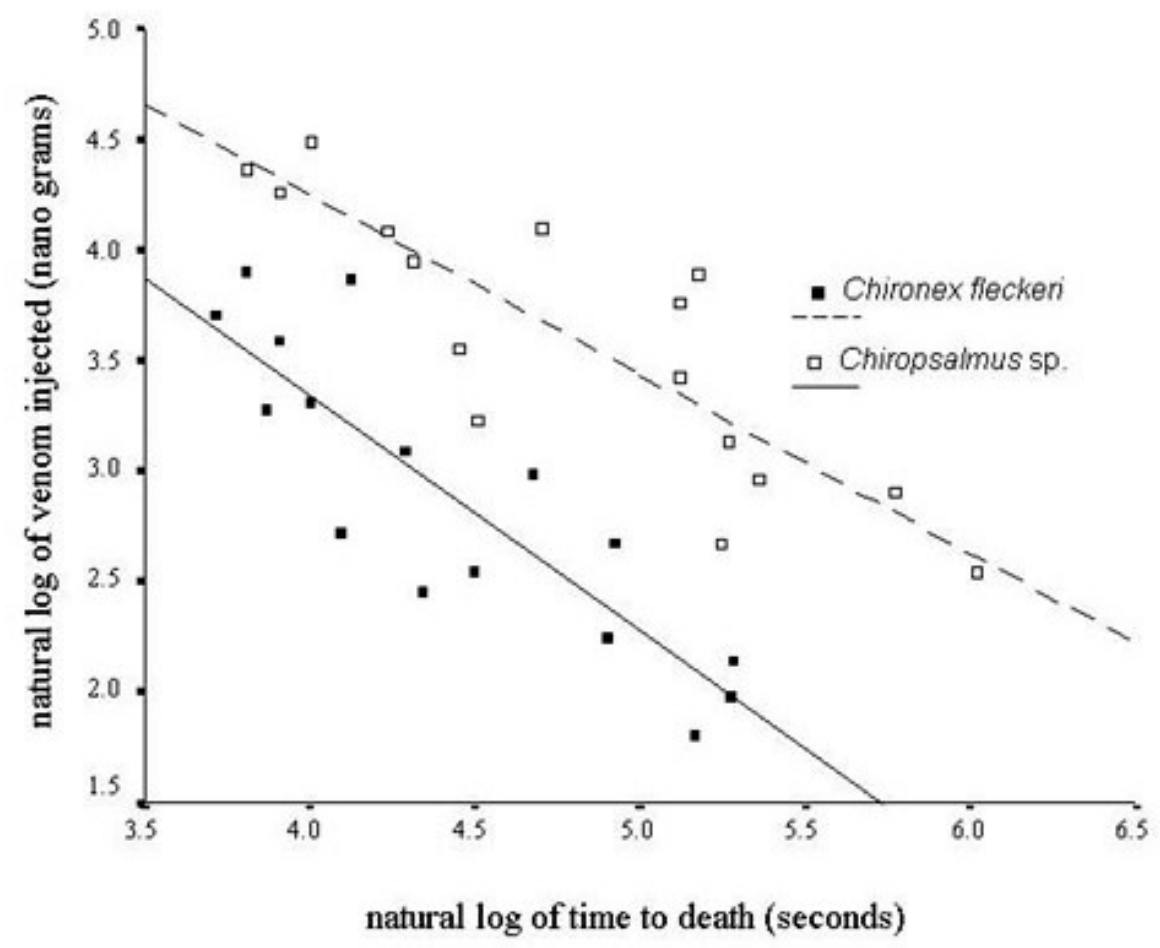

Figure 1: Natural log of venom concentration over natural log of time to death for Chironex fleckeri and Chiropsalmus sp.

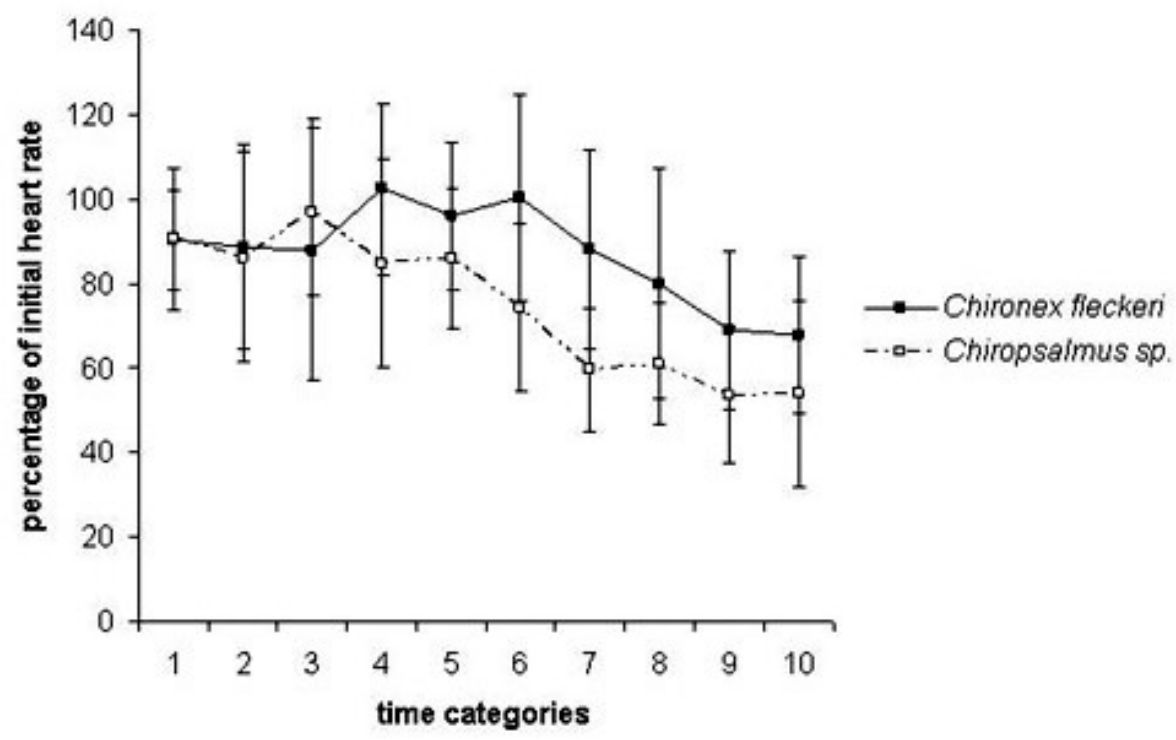

Figure 2: Percentage of initial heart rate over time category for animals envenomed with Chironex fleckeri and Chiropsalmus sp. venom (95\% confidence limits displayed). 


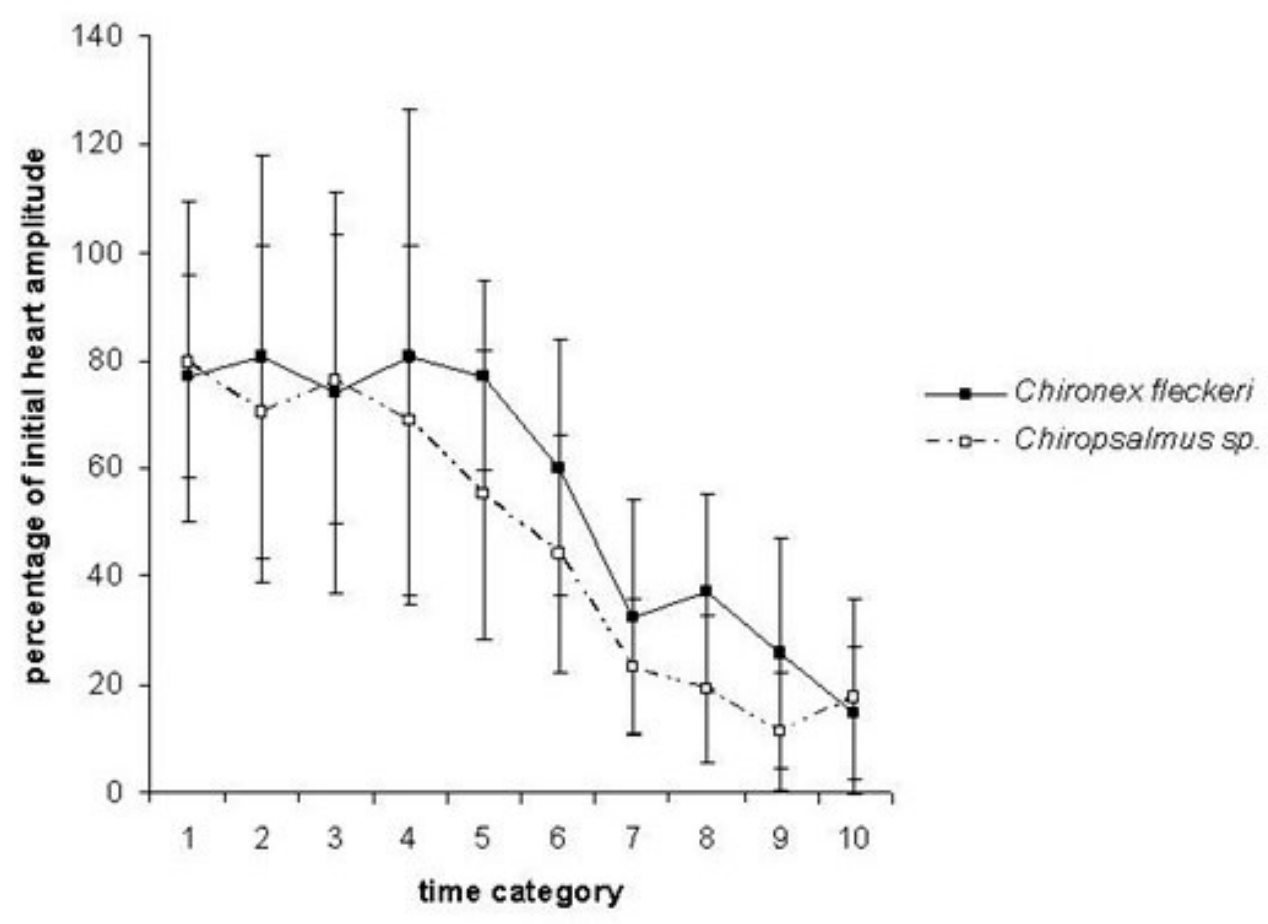

Figure 3: Percentage of initial heart amplitude over time category for animals envenomed with Chironex fleckeri and Chiropsalmus sp. venom (95\% confidence limits displayed).

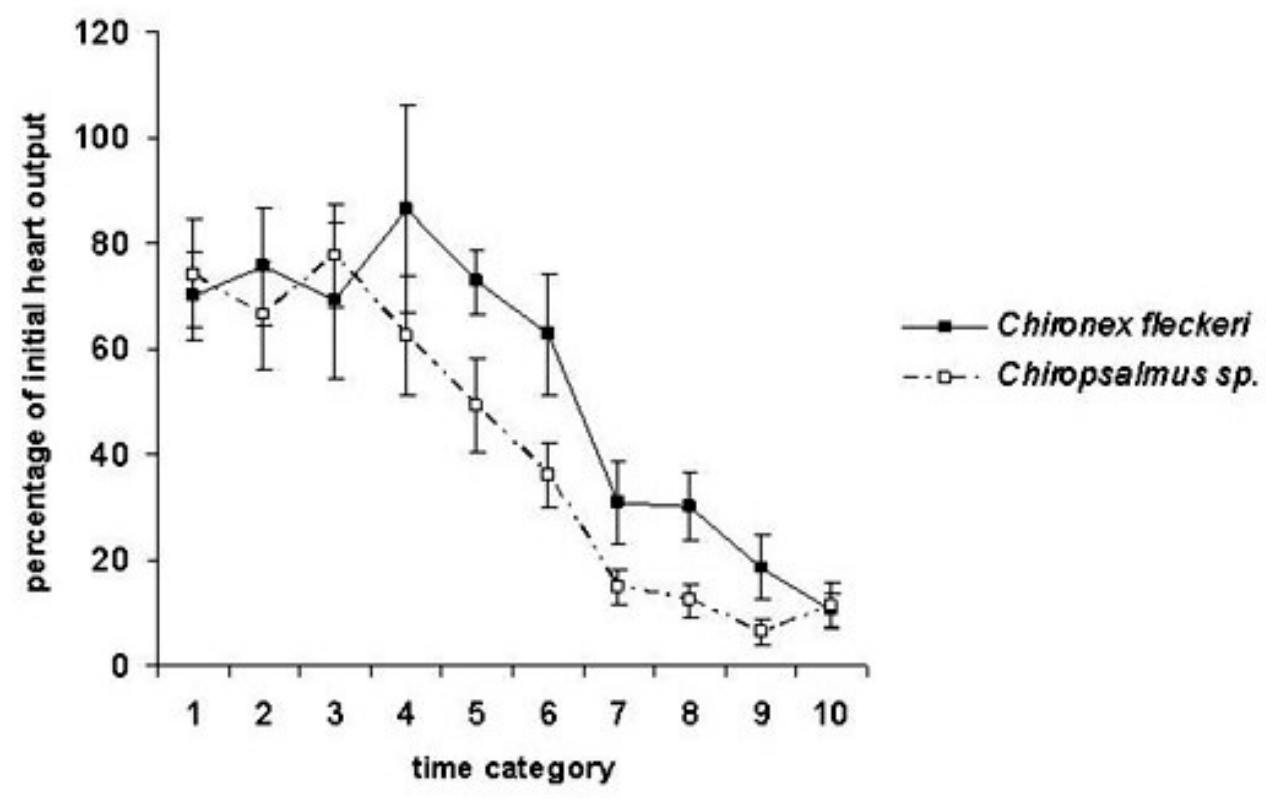

Figure 4: Percentage of initial heart output over time category for animals envenomed with Chironex fleckeri and Chiropsalmus sp. venom (95\% confidence limits displayed). 


\section{DISCUSSION}

The relative heart rate in experimental animals decreased at a distinctly slower rate after injections of $C$. fleckeri venom than that of animals injected with Chiropsalmus sp. venom. Bradycardia has previously been displayed in whole vertebrate and isolated heart preparations for both these species' venoms $(10,11,13,17)$, however this observation of differences in bradycardia produced by the venom of these two species of cubozoans has not been documented before. The relative amplitude of heart contractions also displayed a more pronounced decrease in animals injected with Chiropsalmus sp. venom compared to those injected with $C$. fleckeri venom. Previous studies have noted the reduction in heart amplitude for vertebrate models and isolated hearts with venom from both these species $(10,13,17)$, however, any comparisons of the two species again has not been documented. Heart rate and heart amplitude change combined are reflected in the overall output or effectiveness of the heart and as was the trend for these two recordings, cardiac output also decreased over time at a relatively greater rate in Chiropsalmus sp. than in C. fleckeri injected specimens.

Higher dosage levels of Chiropsalmus sp. venom are required to produce death in the same amount of time as $C$. fleckeri venom, supporting previous research of reduced rates of toxicity in Chiropsalmus sp. $(13,15)$. However, in regards to the specific action of each venom on the invertebrate model examined, it would appear that as far as effectively reducing or hampering cardiac function, Chiropsalmus sp. venom is more efficient. The functional ability of heart rate and heart amplitude are dramatically hampered after injection of Chiropsalmus sp. venom, with implications for the total cardiac output, much more than in specimens injected with $C$. fleckeri venom. This perhaps is suggestive that the mode of action of these two venoms is different.

In an attempt to isolate the components contributing to cubozoan venoms, various authors utilizing different methods of venom extraction have investigated the extracts molecular weights $(2,3,7,8,9)$. Not only has variation been observed between these two species but also between authors investigating the same species, which has been suggested to be an artefact of the different extraction methods utilized and the exposure of venom to different media after extraction (9). 
One study investigating the composition of both species of venom however, using the same extraction method, found a distinctly higher number of protein components [20] in C. fleckeri venom compared to that of Chiropsalmus sp. [8] (16). Within this study, investigation into the components of these venoms using FPLC and SDS-PAGE analysis has indicated that the prominent proteins in these two species of cubozoans are also dramatically different in size (16). Taking these variations into account and those of the difference in nematocyst composition displayed between species (4), it is not surprising that venom extracted from these two species should display a variation in response when comparisons are made on the same whole animal model. As these venoms are quite complex in nature with a number of different protein components, a number of different processes contributing to prey death may be occurring. Some form of amplitude reduction is displayed from both venoms, however the heart rate variation between species of venoms may indicate a difference in the target actions on this invertebrate heart.

As these two species have distinctly different prey types when mature, but similar prey types when young, with Chiropsalmus sp. feeding on invertebrates and $C$. fleckeri shifting to a predominance of vertebrate prey (4), it is perhaps not surprising that the species observed to be predominantly an invertebrate feeder (Chiropsalmus $\mathrm{sp}$.) is more efficient in inhibiting the cardiac function of an invertebrate heart.

Future directions for this study would have to include a similar comparison of cardiac action for fish, which feature as the predominant prey for $C$. fleckeri (4). This would give an indication as to whether the reverse action is displayed on such a model, with C. fleckeri more effective in decreasing cardiac function of their predominant vertebrate prey than Chiropsalmus sp. 


\section{REFERENCES}

1 BARNES JH. Studies on three venomous cubomedusae. In: REES WJ. Ed. The cnidaria and their evolution. London: Zoological Society of London, 1966: 30732.

2 BAXTER EH., MARR AGM. Sea wasp (Chironex fleckeri) venom: lethal, haemolytic and dermonecrotic properties. Toxicon, 1969, 7, 195-210.

3 BLOOM DA., BURNETT JW., ALDERSLADE P. Partial purification of box jellyfish (Chironex fleckeri) nematocyst venom isolated at the beachside. Toxicon, 1998, 36, 1075-85.

4 CARRETTE T., ALDERSLADE P., SEYMOUR J. Nematocyst ratio and prey in two Australian cubomedusans, Chironex fleckeri and Chiropsalmus sp. Toxicon, 2002, 40, 1547-51.

5 CARRETTE T., SEYMOUR J. A rapid and repeatable method for venom extraction from Cubozoan nematocysts. Toxicon, 2004, 44, 135-9.

6 CARRETTE T., SEYMOUR J. Vascular dopplers: a new way of recording cardiac parameters in envenomed organisms. Toxicon, 2005, 45, 541-4.

7 CRONE HD., KEEN TE. Chromatographic properties of the hemolysin from the cnidarian Chironex fleckeri. Toxicon, 1969, 7, 79-87.

8 CRONE HD., KEEN TE. Further studies on the biochemistry of toxins from the sea wasp Chironex fleckeri. Toxicon, 1971, 9, 145-51.

9 ENDEAN R. Separation of two myotoxins from nematocysts of the box jellyfish Chironex fleckeri. Toxicon, 1987, 25, 483-92.

10 ENDEAN R., HENDERSON L. Further studies of toxic material from nematocysts of the cubomedusan, Chironex fleckeri Southcott. Toxicon, 1969, 7, 303-14.

11 ENDEAN R., MONKS SA., CAMERON AM. Toxins from the box jellyfish Chironex fleckeri. Toxicon, 1993, 31, 397-409.

12 ENDEAN R., RIFKIN J. Envenomation involving nematocysts of the box jellyfish Chironex fleckeri. Toxicon, 1983, 3, 115-8.

13 FREEMAN SE., TURNER RJ. Cardiovascular effects of cnidarian toxins: a comparison of toxins extracted from Chiropsalmus quadrigatus and Chironex fleckeri. Toxicon, 1972, 10, 31-7.

14 HAECKEL E. Das system der medusen. Erster theil einer monographie der medusen. Denksch. Med.-Naturwiss. Ges. Jena., 1879, 1, 361-672. 
T. Carrette and J. Seymour CARDIOTOXIC EFFECTS OF VENOMS FROM Chironex fleckeri AND Chiropsalmus sp. ON AN INVERTEBRATE MODEL. J. Venom. Anim. Toxins incl. Trop. Dis., 2006, 12, 2, p.254

15 KEEN TE. Comparison of tentacle extracts from Chiropsalmus quadrigatus and Chironex fleckeri. Toxicon, 1971, 9, 249-54.

16 OLIVER LM. A cubozoan study - a comparison of cnidomes, venom and nematocyst inhibition. Townsville: James Cook University, School of Tropical Biology, 1999. 89f. Thesis [B. Sc. Honours]

17 TURNER RJ., FREEMAN SE. Effects of Chironex fleckeri toxin on the isolated perfused guinea pig heart. Toxicon, 1969, 7, 277-86. 